\title{
NGAL and early AKI detection, call for action!
}

Abbreviations: AKI, acute kidney injury; SCr, serum creatinine; ATN, acute tubular necrosis; NGAL, neutrophil gelatinaseassociated lipocalin; DGF, delayed graft function.

\section{Editorial}

Acute Kidney Injury (AKI) is defined as rapid reduction in kidney function because of multiple causes. Its incidence is increasing in hospitalized patients, especially in critical illness. Worldwide AKI incidence was estimated to be $21.6 \%$ in adults and $33.7 \%$ in children. ${ }^{1}$ AKI is diagnosed only when significant nephron damage occurs, serum creatinine ( $\mathrm{SCr}$ ) rises $\geq 0.3 \mathrm{mg} / \mathrm{dL}$ or oliguria is present. It remains important to recognize the limitations imposed by the use of $\mathrm{SCr}$ and urine output for the detection of AKI. SCr concentration may increase slowly, perhaps only following a major kidney damage. $\mathrm{SCr}$ is also influenced by factors such as age, gender, and muscle mass and nutritional status. It is also unable to differentiate pre-renal AKI from intrinsic renal AKI like acute tubular necrosis (ATN). AKI should be diagnosed earlier in order to protect against adverse outcomes. There is increasing evidence for adverse outcomes associated with AKI including, longer hospital length of stay, significant complication rates (including infection), risk of $\mathrm{CKD}$, development of cardiovascular disease and higher mortality.

The risk of worsening or de novo CKD following an episode of AKI (even if renal functions returned back to normal) remains high. ${ }^{2}$ Fast and accurate AKI biomarkers will significantly improve morbidity and mortality by providing early diagnosis. This is particularly important for effective patient management in AKI. Neutrophil gelatinaseassociated lipocalin (NGAL) seem to represent a suitable option to accomplish this task and replace SCr in AKI diagnosis. Neutrophil gelatinase-associated lipocalin (NGAL) is a $25-\mathrm{kDa}$ glycoprotein produced by epithelial tissues in several organs. NGAL is excreted via glomerular filtration and completely reabsorbed by tubular cells. Urinary NGAL is sensitive and specific for early ATN (not increased in pre-renal AKI). It rises 2-4h post-AKI. Plasma NGAL may also help to predict likelihood of renal recovery. NGAL has been validated in different AKI settings including; pediatric AKI, Sepsis-induced AKI, Delayed Graft Function in Kidney Transplantation from Extended Criteria Donors, in fluid resuscitation of severely burned patients and others. ${ }^{3}$

For example, in a cross-sectional study, adults in the intensive care unit with established AKI (defined as a doubling of the SCr) due to sepsis, ischemia, or nephrotoxins displayed a more than 10 -fold increase in plasma NGAL and more than a 100-fold increase in urine NGAL by Western blotting when compared to normal controls. Both plasma and urine NGAL correlated highly with serum creatinine levels. These results identified NGAL as a sensitive biomarker to established AKI in humans. ${ }^{4}$ Also, in children undergoing cardiopulmonary bypass, NGAL measurement by Western blotting and ELISA showed a 10-fold or more increase in the urine and plasma within 2-6 $\mathrm{h}$ of surgery in patients who subsequently developed AKI (diagnosed using SCr after 1-3 days following surgery). Both urine and plasma NGAL were powerful independent predictors of AKI. ${ }^{5}$ Again, NGAL has also been evaluated as a biomarker of delayed graft function (DGF) in kidney transplantation. A significant correlation between NGAL staining intensity (in biopsies obtained one hour after vascular

\author{
Volume 3 Issue 3 - 2016 \\ Mohamed E Elrggal \\ Kidney \& Urology Center, Egypt \\ Correspondence: Mohamed E Elrggal, Kidney \& Urology \\ Center,Alexandria, Egypt, Tel +2-01005038467, \\ Emailm_elrgal@hotmail.com \\ Received: April 28, 2016 | Published: April 29, 2016
}

anastomosis) and the subsequent development of DGF was found. ${ }^{6}$ Benefits of early AKI diagnosis are countless. It allow for therapeutic intervention either by stopping an offending drug or correcting an underlying condition or for example starting a CRRT.

Furthermore, biomarkers like NGAL are able to distinguish causes of AKI (increase in ATN, Normal in pre-renal AKI). Also, therapeutic intervention for early AKI couldn't be applied early except after detecting and defining early AKI even before urine output decreases or creatinine increases. In summary, NGAL is emerging as an early, sensitive biomarker in the field of AKI. Strong evidence has been provided for the predictability of NGAL in a variety of AKI settings. NGAL should start to pave its way into clinical practice (suggested approach for NGAL start of use in clinical practice is summarized in Figure 1). Benefits of such a diagnostic tool will surely exceed the costs for its implementation. And advancements in nephrology practice will certainly take place with the routine use of NGAL.

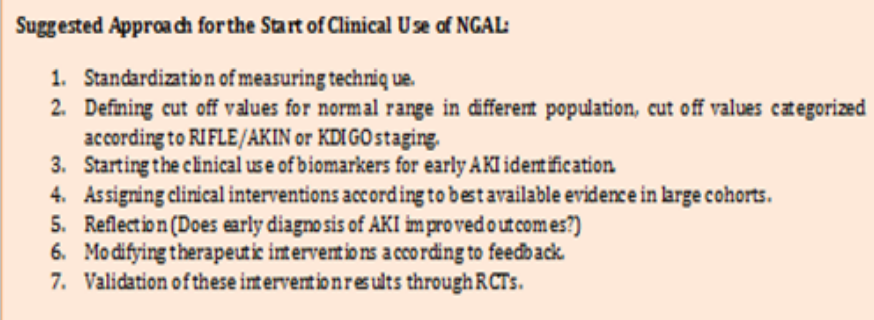

Figure I A suggested approach for NGAL use in clinical practice.

\section{Acknowledgments}

None.

\section{Conflicts of interest}

The author declares there is no conflict of interest.

\section{References}

1. Susantitaphong P, Cruz DN, Cerda J, et al. World incidence of AKI: a meta-analysis. Clin J Am Soc Nephrol. 2013;8(9):1482-1493.

2. Bucaloiu ID, Kirchner HL, Norfolk ER, et al. Increased risk of death and de novo chronic kidney disease following reversible acute kidney injury. Kidney Int. 2012;81(5):477-485. 
3. Ronco C, Legrand M, Goldstein SL, et al. Neutrophil gelatinaseassociated lipocalin: ready for routine clinical use? An international perspective. Blood Purif. 2014;37(4):271-285.

4. Mori K, Lee HT, Rapoport D, et al. Endocytic delivery of lipocalinsiderophore-iron complex rescues the kidney from ischemia-reperfusion injury. J Clin Invest. 2005;115(3):610-621.
5. Mishra J, Dent C, Tarabishi R, et al. Neutrophil gelatinase-associated lipocalin (NGAL) as a biomarker for acute renal injury after cardiac surgery. Lancet. 2005;365(9466):1231-1238.

6. Mishra J, Ma Q, Kelly C, et al. Kidney NGAL is a novel early marker of acute injury following transplantation. Pediatr Nephrol. 2006;21(6): $856-863$. 